\title{
L'UTILISATION DES LAITS TUBERCULEUX (1)
}

\author{
par \\ J. VERGE \\ et \\ G. THIEULIN \\ Professeur à l'Ecole d'Alfort \\ Agrégé des Ecoles vétérinaires
}

En exécution de l'article 7 de la loi du 7 juillet 1933 sur la prophylaxie de la tuberculose des bovidés et sur le contrôle de la salubrité des viandes, le Décret du 24 janvier 1934 stipule en son article 4 :

"Les laits provenant d'animaux atteints de tuberculose et les laits renfermant des bacilles tuberculeux ne pourront être utilisés pour l'alimentation de l'homme et des animaux, soit en nature, soit sous forme de produits dérivés, qu'après un chauffage assurant la destruction du bacille tuberculeux.

(Toutefois, les laits provenant des animaux atteints des formes de tuberculose prévues par l'article 2 de la loi du 7 juillet 1933 devront être détruits dans tous les cas. ”

Cet article 4 du décret du 24 janvier 1934 abroge implicitement l'arrêté ministériel en date du 28 juillet 1898 qui, dans son article 13 , interdisait la vente et l'usage du lait provenant des vaches tuberculeuses et permettait son utilisation sur place, pour l'alimentation des animaux, après avoir été bouilli.

L'arrêté ministériel du 28 juillet 1898 n'avait d'ailleurs jamais reçu la moindre application effective.

Le décret du 24 janvier 1934 rend exécutoires les prescriptions de l'article 2 du décret du 25 mars 1924, portant règlement d'administration publique, pour le lait et les produits dérivés, de la loi du $1^{\text {er }}$ août 1905 sur la répression des fraudes. Dans ces conditions, les pénalités inscrites dans cette loi sont maintenant applicables.

Le nouveau texte présente une importance essentielle. Aussi convient-il de le discuter pour mieux l'interpréter, dissiper toute équivoque et en dégager la portée et les conséquences.

Le décret du 24 janvier 1934, contrairement à l'arrêté du 28 juillet 1898, permet l'utilisation de la p'upart des laits tuberculeux pour l'alimentation de l'homme, sous réserve, bien entendu, de la destruction des bacilles de Koch. De tels liquides redeviennent marchands. Seuls certains laits, suspects d'être fortement contaminés et provenant de vaches atteintes d'une tuberculose légèrement contagieuse, sont exclus de tout commerce et devront être détruits. L'existence des laits tuberculeux est ainsi reconnue par la loi et apparaît dès lors comme un fait inéluctable, dont on ne peut que limiter les fâcheuses conséquences. On doit renoncer raison-

(1) Revue générale de Médecine vétérinaire, t. XLV, n540, 15 décembre 1936, p. 713 . 
nablement, bien que cela soit une mesure idéale, à détruire tous les laits tubereuleux. Ainsi, à l'exclusion des cas graves, les laits tubereuleux, "assainis ", seront consommables. Pour désagréable que soit ce point de vue, il faut en reconnaitre la légitimité.

Sans discuter les faits, leurs causes et leurs remèdes, les nouvelles prescriptions que nous étudions ici ne visent que "les modes d'utilisation" des laits tuberculeux ou soupçonnés de l'être par leur origine - et c'est là leur caractère essentiel.

Cette préoccupation hygiénique qui tend à restreindre l'utilisation des laits tuberculeux - qu'on ne peut économiquement détruire en totalité - est due aux graves dangers qu'ils présentent.

Le pouvoir pathogène pour l'homme du bacille de Koch (type bovin), n'est plus à établir. De plus, les animaux recèlent et excrètent parfois le type humain.

Infiniment plus que l'adulte, l'enfant est vulnérable. Pour établir ce fait, il suffit de relever les nombreux cas de tuberculose infantile dus au bacille de Koch (type bovin) et provoqués, sans aucun doute, par l'ingestion d'un lait bacillifère (1). En effet, le tube intestinal du nourrisson est fragile; le bacille tuberculeux traverse facilement les muqueuses; les ingestions de lait sont fréquentes, souvent plusieurs fois par jour, et longtemps répétées ; la répétition des ingestions s'ajoute ainsi à l'abondance des absorptions : facteurs essentiels. Ces données justifient la sévérité des prescriptions établies.

Toutes les femelles laitières tuberculeuses sont visées par le premier alinéa de l'article 4 du présent décret. Il s'agit donc cette fois non seulement des laits de vache mais encore des laits de chèvre, de brebis, de jument, d'ânesse, etc. Pas de distinction quant à l'espèce. C'est le lait tuberculeux en général qui est visé, à quelque femelle qu'il appartienne. En revanche, le second alinéa ne concerne que les laits provenant des animaux atteints des formes de tuberculose prévues par l'article 2 de la loi du 7 juillet 1933, e'est-à-dire uniquement les vaches laitières. Ainsi, il faut comprendre que tous les laits provenant d'animaux tuberculeux devront être assainis avant de pouvoir être livrés à la consommation, à l'exception de certains laits de vache qui, étant donné la gravité de leur infection, seront détruits. L'alinéa 2 est restrictif et intéresse un cas particulier.

Les laits dont le mode d'utilisation est réglementé sont d'abord ceux "provenant d'animaux atteints de tuberculose». Cette désignation générale englobe tout de suite un lot important. En principe, quand la mamelle est saine, c'est-à-dire exempte de lésions

(1) Voir à ce propos: H. LACHMANa, Bulletin de l'Office International des Epizooties, 1936, t. XIII, p. 122. 
tuberculeuses, même microscopiques, elle constitue une barrière et un filtre infranchissables. Il peut paraître qu'il eût dès lors suffi de eonsidérer seulement les laits issus de mamelles tuberculeuses. En réalité, cette restriction eut été grave de conséquences. Une mamelle saine donne un lait sain, à condition toutefois que ce lait soit recueilli avec grand soin. Une femelle laitière atteinte de tubereulose légère sans signes cliniques et sans lésion mammaire, peut éliminer par son tube digestif des bacilles de Koch. La moindre parcelle de matières fécales est susceptible de contaminer gravement le lait. De plus, si les tuberculoses mammaires sont assez rares, les animaux tuberculeux sont très nombreux. A tout moment peut se produire une lésion microscopique de l'organe sécrétoire : le filtre est troué, le lait va contenir des bacilles de Koch. La menace est permanente.

Il est done prudent de déclarer dangereux, s'il est consommé cru, tout lait provenant d'un animal tuberculeux.

Doit être déclaré atteint de tuberculose tout anímal qui présente une preuve objective et tangible de l'affection, signe clinique, réaction positive à l'épreuve de la tuberculine, etc.

En second lieu, sont également visés "les laits renfermant des bacilles tuberculeux ". C'est qu'en effet des laits ne provenant pas d'animaux atteints de tuberculose peuvent être secondairement infectés par mélange avec un lait bacillifère. Ils peuvent être infectés également par le trayeur, par les divers manipulateurs, par des excréteurs de bacilles; ils peuvent être souillés pär les récipients au cours des transvasements, etc. Mais alors, comment reconnaître ces laits en temps utile? On peut dire, d'une façon générale, que tout lait de mélange risque fort de contenir des bacilles de Koch.

L'examen clinique et la pratique de la tuberculine permettent de constituer trois catégories d'animaux :

a) Les animaux atteints d'une forme de tuberculose réputée légalement contagieuse ;

b) Les animaux tuberculeux autres que les précédents;

c) Les animaux indemmes de tuberculose.

Le lait des animaux appartenant au second groupe doit être assaini. Les sujets sains donneront un lait non infecté à l'origine, c'est-à-dire dans la mamelle. Mais comment garantir qu'il n'y aura pas pollution du lait après la traite et que, partant, ce lait ne renfermera pas à un moment donné les germes spécifiques?

Ou bien nul compte ne sera tenu de la réglementation visant « ces laits renfermant des bacilles tuberculeux " et ce sera très grave, ou bien il faudra assainir tous les laits, sauf ceux qui, provenant d'animaux non tuberculeux, seront recueillis et transportés dans des 
conditions telles que leur infection secondaire - par mélange par exemple avec des laits anonymes - ne sera pas possible. Cette variété particulière de lait non tuberculeux se confondra alors avec la variété créée par le contrôle officiel facultatif, variété actuellement exceptionnelle.

L'arrêté du 28 juillet 1898 interdisait l'utilisation des laits tuberculeux pour l'alimentation de l'homme. Il ne l'autorisait que pour l'alimentation des animaux, après ébullition. L'article 4 du décret du 24 janvier 1934 étend à l'alimentation de l'homme l'usage de ces laits, mais sous certaines conditions.

Les laits tuberculeux sont également dangereux pour l'homme et pour les animaux. Les pores alimentés avec des laits écrémés et des 'sérums de fromagerie présentent souvent des localisations tuberculeuses qui traduisent et mettent en lumière le mode d'infection (tuberculose des premières voies digestives provoquée par l'ingestion répétée de produits bacillifères). Des chevaux ont été trouvés, en Angleterre, porteurs de bacilles bovins, ce fait étant dû à l'ingestion répétée de laits infectés. Enfin, d'un point de vue plus général, il est normal que les mesures énoncées soient étendues à tous les consommateurs de lait sans distinction d'espèce - ce qui permet de faire œuvre logique et judicieuse de prophylaxie.

Une précision fort importante est apportée par les termes «soit en nature, soit sous forme de produits dérivés ». Les crèmes et les beurres fabriqués à partir de laits tuberculeux sont virulents. Les fromages même fermentés, restent dangereux pendant plusieurs semaines. Mais cette interdiction, dictée par l'hygiène, d'utiliser après assainissement des laits tuberculeux pour la fabrication des produits dérivés pose un problème économique et technique de réelle importance.

L'assainissement, c'est-à-dire le chauffage suffisant des laits et des crèmes, permet la fabrication des beurres. Bien que la quantité de beurres préparés au moyen de crèmes non chauffées soit encore importante, rien n'empêche l'observation immédiate, hautement souhaitable, du récent décret. La même méthode ne saurait valoir pour tous les laits destinés à la fromagerie (à l'exception des fromages frais), du moins pour l'instant. Pour pouvoir appliquer à la lettre les nouvelles prescriptions, il faut que les techniques fromagères actuelles subissent des transformations, disons des bouleversements. Il conviendrait donc, en attendant, d'indiquer des mesures préventives et de fixer les modalités à suivre aux lieux de production et au cours des manipulations pour, d'une part, ne pas produire de laits tuberculeux et, d'autre part, ne pas contaminer le lait sain pendant ou après sa récolte. 
La méthode d'assainissement fixée, est un " ehauffage assurant la destruction du bacille de Koch ".

L'action de la chaleur constitue, à l'heure actuelle, la seule technique souhaitable. L'addition de produits chimiques est interdite en France dans les substances alimentaires ; à plus forte raison, la stérilisation chimique n'est-elle pas tolérée. Quant aux méthodes autres que le chauffage, il n'en existe pas qui puisse offrir la moindre garantie.

Le terme chauffage est général. Tout procédé utilisant la chaleur comme agent de destruction pourra être accepté si la technique mise en œuvre assure la destruction certaine des bacilles de Koch. C'est très bien ainsi. On ne saurait en effet, au nom de l'hygiène, imposer tel procédé particulier. L'ébullition est un mode domestique; la pasteurisation et la stérilisation sont des modes industriels. Parmi ces derniers, la pasteurisation est à la fois le procédé le plus courant et le plus simple permettant l'établissement des techniques les plus variées. Qu'il s'agisse de pasteurisation basse, de pasteurisation en couche mince, de pasteurisation haute, ce qui importe ici c'est la destruction des bacilles de Koch. Or, à l'heure actuelle, de nombreux procédés donnent de suffisantes garanties, à condition que les techniques reconnues bonnes soient rigoureusement observées et surveillées (degré et durée de chauffage) et que les défaillances possibles des appareils soient évitées par un fonctionnement sévèrement contrôlé. Bien entendu, l'opération devra comporter une suite propre à éviter toute infection ultérieure.

Ainsi que nous l'avons dit plus haut, le deuxième alinéa de l'article 4 du décret du 24 janvier 1934 est restrictif et vise le cas particulier des laits provenant de vaches laitières atteintes de «tubereulose avancée du poumon, de tuberculose de l'intestin, de la mamelle ou de l'utérus ".

La sévérité de ce deuxième alinéa n'a rien d'excessif si l'on tient compte de la portée générale que doit avoir la loi du 7 juillet 1933 sur la prophylaxie de la tuberculose. Les bovins tuberculeux atteints des formes précitées doivent disparaître le plus rapidement possible de l'exploitation. Ils constituent des sources d'infection ; ils assurent la pérennité du contage ; ils représentent véritablement des "non valeurs économiques", ce sont des animaux hors commerce. Leur lait est le plus souvent fortement contaminé (surtout dans le cas de tuberculose mammaire, de tuberculose intestinale et utérine). Celui-ci ne saurait devenir, même après chauffage efficace, un aliment sain, ni logiquement constituer une source de revenus.

Eliminant du commerce les animaux d'espèce bovine atteints de ces formes graves de tuberculose, les séquestrant et tendant à leur disparition, il est logique - en dehors de tout autre point de vue - 
d'ordonner la destruction de leur lait, ce qui permet de faire ouvreessentielle de prophylaxie.

Le décret du 24 janvier 1934 précise ainsi les conditions d'utilisation des laits tuberculeux ou soupçonnés de l'être. Quelles sanctions seront applicables alors à la non observation des règles ainsi établies ?

\section{L'article 3 du décret du 25 mars 1924 énonce :}

"Est considéré comme une tentative de tromperie, aux termes de l'article premier de la loi du 1 er août 1905, le fait de détenir sans motifs légitimes, d'exposer, de mettre en vente ou de vendre pour la consommation humaine du lait impropre à cet usage. "

Or, tout lait provenant d'animaux tuberculeux ou renfermant des bacilles de Koch et n'ayant pas subi un chauffage assurant la destruction de ces germes, est impropre à l'alimentation de l'homme. De même - et ici dans tous les cas - pour les laits provenant des animaux atteints des formes de tuberculose prévues à l'article 2 de la loi du 7 juillet 1933. Rappelons également le deuxième alinéa de

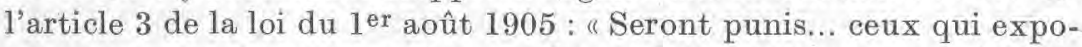
seront, mettront en vente ou vendront des denrées servant à l'alimentation de l'homme ou des animaux..., des produits..., qu'ils sauront être falsifiés, corrompus ou toxiques. »

Tout ceci montre la sévérité des peines encourues.

Ces points importants étant acquis, il convient d'envisager les modalités d'application des mesures édictées.

Quatre variétés de lait sont à considérer :

A. Laits provenant d'animaux non tuberculeux et ne subissant pas d'infection secondaire avant d'atteindre le consommateur ;

B. Laits provenant d'animaux tuberculeux non contagieux au sens de la loi ; Koch.

C. Laits contenant ou pouvant contenir des bacilles de

D. Laits provenant d'animaux atteints d'une forme de tuberculose réputée légalement contagieuse.

La vente des laits de la catégorie A - rares, pour ne pas dire exceptionnels - est libre.

La destruction des laits de la catégorie D est obligatoire.

Les deux catégories $\mathrm{B}$ et $\mathrm{C}$ englobent la grosse majorité des laits du commerce. Ces laits ne pourront être vendus qu'après assainisse- 
ment par chauffage. Il s'agit pratiquement de la pasteurisation obligatoire.

Pour veiller à l'observation du décret du 24 janvir 1934 et garantir l'application des graves mais salutaires sanctions prévues, rappelons qu'il faut absolument instituer :

$1^{\circ}$ Le contrôle hygiénique du lait à la production - ce qui permettra d'éliminer réellement les laits hors commerce en dépistant les vaches laitières atteintes de tuberculose avancée du poumon, de tuberculose mammaire, intestinale ou génitale ;

$2^{\circ}$ Le contrôle hygiénique du lait à la consommation qui comprendra, pour les fins précises poursuivies ici, la contrôle rigoureux que réalise l'inspection bactériologique.

\title{
L'ÉPURATION DU LAIT DANS L'INDUSTRIE LAITIÈRE. NÉCESSITÉ DE LA FILTRATION OU DE L'ÉPURATION CENTRIFUGE. AVANTAGES ET INCONVÉNIENTS (1)
}

\author{
par J. GIROUX
}

Ingénieur Agronome. Directeur du Laboratoire central du contrôle des laits et de recherches chimiques et bactériologiques de la Société Laitière MAGGI.

Dans un grand nombre d'exploitations agrieoles, l'étable est mal aménagée ; l'état de propreté du sol, des murs et des litières est pitoyable ; les animaux producteurs sont souillés d'excréments.

Les soins apportés à la traite sont insuffisants. La mamelle n'est pas lavée. Seule, l'heure de la mulsion est à peu près réglée, mais aucune précaution n'est prise pour éviter les contaminations du précieux liquide alimentaire que nous aurons plus tard à assainir et à transformer.

Il est même d'une pratique courante qu'on affourage le bétail pendant que le vacher procéde à la traite.

Ces fautes et négligences si préjudiciables à la qualité et à l'hygiène du lait, dès la production, occasionnent toujours de lourdes pertes pour l'industrie et ces pertes se traduisent finalement par des prix moins rémunérateurs offerts au cultivateur pour l'achat de ses fournitures. D'autre part, le développement de la consommation est également affecté par cette répugnance légitime "du lait sale ", et cette mévente est d'autant plus regrettable qu'elle coïncide, dans notre pays, avec une production surabondante qui atteint déjà 150, millions d'hectolitres par an.

Nous savons bien qu'il est difficile d'obtenir le refroidissement du lait à la ferme. En différents points de notre territoire, les exploi-

(1) Rapport présenté au Congrès national de l'Industrie laitière. Nancy, 24-25 sepembre 1936. 